\title{
Diagnostic utility of 3D DIR MRI in the estimation of MS lesions overall load with special emphasis on cortical subtypes
}

\author{
Ahmed S. Abdelrahman * ${ }^{*}$, Nivan Hany Khater and Mai M. K. Barakat
}

\begin{abstract}
Background: A number of magnetic resonance imaging studies have shown that a significant proportion of multiple sclerosis (MS) lesions are located in the cerebral cortex and correlate with the patient's disability level. Double inversion recovery (DIR) has a higher sensitivity to detect cortical lesions and their different types. The aim of our study was to assess the superiority of 3D DIR as compared to FLAIR in detecting the different cortical subtypes and to enhance its role as a test for the clinical disability of the patient.

Results: The current study included 82 MS patients (60 female and 22 male with mean age of $34.5 \pm 8.2$ years); 59 cases having relapsing-remitting MS, 5 having secondary progressive MS, and 4 having primary progressive MS, as well as eight clinically isolated syndrome cases, and six radiologically isolated syndrome cases. DIR was superior to FLAIR in MS plaque number detection of cortical plaque-types 1, 2, 3 and 4 with a $p$ value $<0.001$. DIR was superior to FLAIR for detection of overall cortical types, juxtacortical and juxtacortical extended MS plaques with $p=<0.001$, 0.010 and $<0.001$, respectively. DIR was significantly better than FLAIR for MS plaque number detection in the periventricular white matter, subcortical white matter, infratentorial region and the overall MS plaques burden with a $p<0.001,0.038,<0.001,<0.001$, respectively. No significant difference was noted between both sequences for plaques number detection in deep white matter and deep gray matter. A significant correlation between MS disease duration, number of attacks and EDSS and the overall cortical MS plaques types and the overall MS plaque burden.
\end{abstract}

Conclusion: 3D DIR is a reliable tool and provides superior delineation in detecting cortical subtypes. In addition, DIR is of value in assessing the degree of clinical and cognitive impairment of MS affected patients.

Keywords: DIR, MRI, MS, Cortical plaques, Subtypes

\section{Background}

Over the past years, a number of magnetic resonance imaging (MRI) studies have provided clear evidence that the earliest stages of multiple sclerosis (MS) disease show damage and atrophy of the gray matter even faster than that of white matter atrophy [1]. The severity of cortical inflammation shows a good correlation with the rate at which the disease progresses [2]. Recent studies suggested that the cortical lesions (CLs) show active

\footnotetext{
*Correspondence: dr_ahmedsamy@yahoo.com

Radiology Department, Faculty of Medicine, Ain Shams University, Cairo, Egypt
}

neuroinflammation, and the histopathology of these lesions show extensive amounts of inflammatory components as T-cell infiltrates, B-cell follicle-like structures, lymphocytes and macrophages and conglomerates of microglia [3].

Cortical lesions have, thus, been included in the recent 2017 revised Mcdonald's diagnostic criteria for MS due to their specificity and pivotal role in the pathophysiology of MS [4]. Cortical lesions may remain undetected with conventional imaging sequences as $\mathrm{T} 2$ and FLAIR, yet it is more discernible and clearly seen with the double inversion recovery (DIR) sequence [5]. DIR is a special pulse sequence that nulls the signal 
from cerebrospinal fluid (CSF) and white matter of the brain making it easier to detect cortical lesions and useful to detect MS at its earliest stages [6].

The expanded disability status scale (EDSS) is an important 20-step scale used nowadays to assess the degree of disability and severity ranging from 0 (normal) to 10 (death due to MS) [7]. Proper estimation of the gray matter lesion load is essential to assess both neuropsychological and physical deficits of MS patients [8], and it was suggested that the degree of disability progression in MS positively correlates with cortical lesions and is partially independent of subcortical white matter pathology [2].

A cortical lesion classification has been accepted with four different types. Type I lesions involve both the white and gray matter, whereas type II lesions are entirely incorporated within the cerebral cortex with no contact with the subcortical white matter or the pia mater, and are small and perivascular. Type III lesions are subpial areas of demyelination that usually do not extend beyond the cortex's layers 3 and 4. Type IV lesions span the entire width of the cortex but don't spread into the subcortical white matter [9].

\section{Aim of the work}

The aim of this study was to assess the accuracy of 3D DIR in delineating the different cortical subtypes and the impact of DIR in evaluating the degree of disability in MS affected patients.

\section{Methods \\ Patients}

This prospective study was conducted in our institution from August 2020 to September 2021, following approval by the institutional ethical committee, with written informed consent signed by all participants.

The current study included 82 patients with MS who were diagnosed using the 2017 revised McDonald criteria [4] and underwent an MRI brain scan that included both FLAIR and 3D DIR sequences. Patients with MRI contraindications, such as non-MRI compatible pacemakers, and those lacking either the FLAIR or DIR sequence were excluded from this study. The current study included 59 cases of relapsing-remitting MS, 5 cases of secondary progressive MS, and 4 cases of primary progressive MS, but there were also eight clinically isolated syndrome cases and six radiologically isolated syndrome cases based on the type of MS at first diagnosis. The EDSS was used to assess patient disability, which ranged from 0 (no disability) to 10 points (patient death due to MS).

\section{Technique of MRI examination}

A 1.5T Philips Achieva MRI unit with a 16-channel sensitivity-encoding head coil was used for the MRI examination. Axial T1WI, axial FLAIR, sagittal FLAIR, axial T2WI and diffusion-weighted imaging were among the standard sequences. Sagittal and axial 3D DIR were obtained in a separate sequence. All patients were examined in the supine position, head first. Acquisition parameters: T1WI: TE15 ms, TR: $450 \mathrm{~ms}$, FOV $230 \times 177 \mathrm{~mm}^{2}$, matrix $208 \times 134$, slice thickness $6 \mathrm{~mm}$. T2WI: TE $100 \mathrm{~ms}$, TR $3612 \mathrm{~ms}$, FOV $230 \times 177 \mathrm{~mm}^{2}$, matrix $208 \times 127$, slice thickness $6 \mathrm{~mm}$. FLAIR: TE $120 \mathrm{~ms}$, TR $6000 \mathrm{~ms}$, TI $2000 \mathrm{~ms}$, FOV $230 \times 184 \mathrm{~mm}^{2}$, matrix $240 \times 111$, slice thickness $6 \mathrm{~mm}$. The scan time of the conventional MRI was $8 \mathrm{~min}$ and $55 \mathrm{~s}$. 3D sagittal DIR: TE $301 \mathrm{~ms}$, TR $5500 \mathrm{~ms}$, TI1 $2510 \mathrm{~ms}$, matrix $164 \times 162$ FOV $230 \times 123 \mathrm{~mm}^{2}$, turbo factor 208 , voxel size $1.34 \times 1.36 \times 0.76 \mathrm{~mm}^{3}$, scan time $3 \mathrm{~min}$ and $23 \mathrm{~s}$. Axial DIR was created from 3D sagittal by MPR.

\section{Analysis of data \\ Imaging analysis}

The FLAIR and 3D DIR sequences were blindly and independently interpreted by two radiologists with 9 and 10 years of experience in neuroimaging, and a final diagnosis was reached by consensus in cases of disagreement.

The MS lesion was identified as hyper-intense focus with a size of $\geq 3 \mathrm{~mm}$. The MS lesions were counted and subsequently classified according to the following anatomical regions: Cortical lesions: which were further classified into 4 types according to $\mathrm{B} \varnothing$ et al. [9] classification: cortical lesion type I; lesion extends through both white matter and gray matter (cortical-juxtacortical lesions), cortical lesion type II; lesion within the cerebral cortex and does not extend to the surface of the brain or the subcortical white matter, cortical lesion type III; subpial lesion, and cortical lesion type IV; lesion extends throughout the full width of the cerebral cortex but does not reach the subcortical white matter. Juxtacortical lesions (JX) which are in the white matter and touch the cortex, Juxtacortical expanded; involving both cortical and juxtacortical lesion, subcortical white matter; lesion in subcortical white matter and don't touch the gray matter, periventricular lesions; lesion touching the lateral ventricles, deep white matter (DWM) lesions which are located in deep white matter, the deep gray matter (GM) lesions which are located in the deep nuclei and finally the infratentorial lesions.

\section{Statistical analysis}

Data were analyzed using Statistical Package for Social Science (IBM Corp. Released 2017. IBM SPSS 
Statistics for Windows, Version 25.0. Armonk, NY: IBM Corp.). Parametric quantitative data were expressed as mean \pm standard deviation (SD). The non-parametric quantitative results were reported using the median and range. Frequency and percentage were used to describe qualitative data. To compare the number of lesions detected by different MRI sequences, a Wilcoxon matched-pairs signed-rank test was used, and the Pearson Chi-Square test was used to compare the number of patients with MS plaques affection detected by the MRI sequence. Spearman correlation was used to examine the relationship between the number of MS plaques and the clinical data of the patients. Statistical significance was defined as a $p$ value of less than 0.05 .

\section{Results}

The current study involved 82 patients with MS, the most common type of MS was relapsing-remitting (59/82; $72 \%)$ followed by secondary progressive $(5 / 82 ; 6.1 \%)$, and primary progressive $(4 / 82 ; 4.9 \%)$, the remaining $(14 / 82$; $17.1 \%)$ was clinically isolated syndrome $(8 / 82 ; 9.8 \%)$ and radiologically isolated syndrome and $(6 / 82 ; 7.3 \%)$ which was proved with follow up to be MS. The mean patient age was $34.5 \pm 8.2$ years with $60(73.2 \%)$ females and 22 (26.8\%) males, the median of the disease duration, number of attacks per year and EDDS was 4, 2 and 2, respectively (Table 1).

Table 2 revealed that the number of MS plaques detected by 3D DIR sequence were significantly more than those detected with FLAIR sequence in cortical plaque-type 1, 2, 3 and 4 with 185\%, 234\%, 258\% and $152 \%$ improvement in the number of the detected MS plaque $(p<0.001)$, respectively, also the 3D DIR was significantly

Table 1 The demographic and clinical data of MS patients

\begin{tabular}{ll}
\hline $\begin{array}{l}\text { Age }(\text { year) } \\
\text { Gender }\end{array}$ & $34.5 \pm 8.2(18-53)$ \\
Female & \\
Male & $60(73.2 \%)$ \\
Duration (year) & \\
Number attacks per year $^{3}$ & $22(26.8 \%)$ \\
EDSS $^{3}$ & $4(3-7)$ \\
Type of MS at first diagnosis $^{2}$ & $2(1.75-4)$ \\
Relapsing-remitting & $2(1-3)$ \\
Secondary progressive & \\
Primary progressive syndrome & $59(71.95 \%)$ \\
Clinically isolated syndrome & $5(6.1 \%)$ \\
Radiologically isolated & $4(4.9 \%)$ \\
\hline
\end{tabular}

\footnotetext{
${ }^{1}$ Mean \pm SD (Range)

${ }^{2}$ Number (\%)

${ }^{3}$ Median (IQR)
}

Table 2 MS plaques load in the different anatomical regions by 3D DIR and FLAIR sequences

\begin{tabular}{|c|c|c|c|c|c|}
\hline $\begin{array}{l}\text { Anatomical } \\
\text { region }\end{array}$ & 3D DIR & FLAIR & $\begin{array}{l}\% \\
\text { improvement }\end{array}$ & z & $p$ value \\
\hline Cortical type I & 293 & 158 & 185 & -6.885 & $<0.001^{*}$ \\
\hline Cortical type II & 356 & 152 & 234 & -6.767 & $<0.001^{*}$ \\
\hline Cortical type III & 31 & 12 & 258 & -3.578 & $<0.001^{*}$ \\
\hline Cortical type IV & 73 & 48 & 152 & -3.917 & $<0.001^{*}$ \\
\hline $\begin{array}{l}\text { Overall cortical } \\
\text { types }\end{array}$ & 753 & 370 & 204 & -7.612 & $<0.001^{*}$ \\
\hline Juxtacortical & 306 & 257 & 119 & -2.570 & $0.010^{*}$ \\
\hline $\begin{array}{l}\text { Juxtacortical } \\
\text { Expanded }\end{array}$ & 1059 & 627 & 169 & -7.562 & $<0.001^{*}$ \\
\hline $\begin{array}{l}\text { Periventricular } \\
\text { WM }\end{array}$ & 925 & 804 & 115 & -4.311 & $<0.001^{*}$ \\
\hline Deep WM & 1100 & 1072 & 103 & -0.947 & 0.344 \\
\hline Subcortical WM & 348 & 311 & 112 & -2.074 & $0.038^{*}$ \\
\hline Infratentorial & 282 & 215 & 131 & -5.446 & $<0.001^{*}$ \\
\hline Deep gray matter & 145 & 128 & 113 & -1.627 & 0.104 \\
\hline $\begin{array}{l}\text { Overall MS } \\
\text { burden }\end{array}$ & 3859 & 3157 & 122 & -7.659 & $<0.001^{*}$ \\
\hline
\end{tabular}

${ }^{*}$ Significant

superior than FLAIR for overall cortical types, juxtacortical, juxtacortical extended MS plaques with 204\%, 119\% and $169 \%$ plaque gain in the 3D DIR relative to FLAIR, respectively ( $p=<0.001,0.010$ and $<0.001$, respectively). Moreover, the 3D DIR was significantly better than FLAIR for MS plaque number detection in the periventricular white matter, subcortical white matter, infratentorial region and the overall MS plaques burden with a relative improvement of $115 \%, 112 \%, 131 \%$ and $122 \%$, respectively $(p<0.001,0.038,<0.001,<0.001$, respectively). On the other hand, no significant difference in the number of detected plaques between both sequences in the deep white matter $(p=0.344)$ and deep gray matter MS plaques ( $p=0.104)$ (Figs. 1, 2, 3, 4, 5, 6).

Table 3 revealed a significant correlation between MS disease duration, number of attacks and EDSS and the overall cortical MS plaques types, as well as the juxtacortical expanded, periventricular white matter, and the overall MS plaque burden, on the other hand, no significant correlation noted for deep white matter and infratentorial MS plaques.

\section{Discussion}

The development of cortical lesions in MS seems to correlate with the clinical course and the degree of patients' disability independent from white matter (WM) evolution [10]. More attention has been drawn to the function of the gray matter and its involvement in the early stages of MS [11]. 


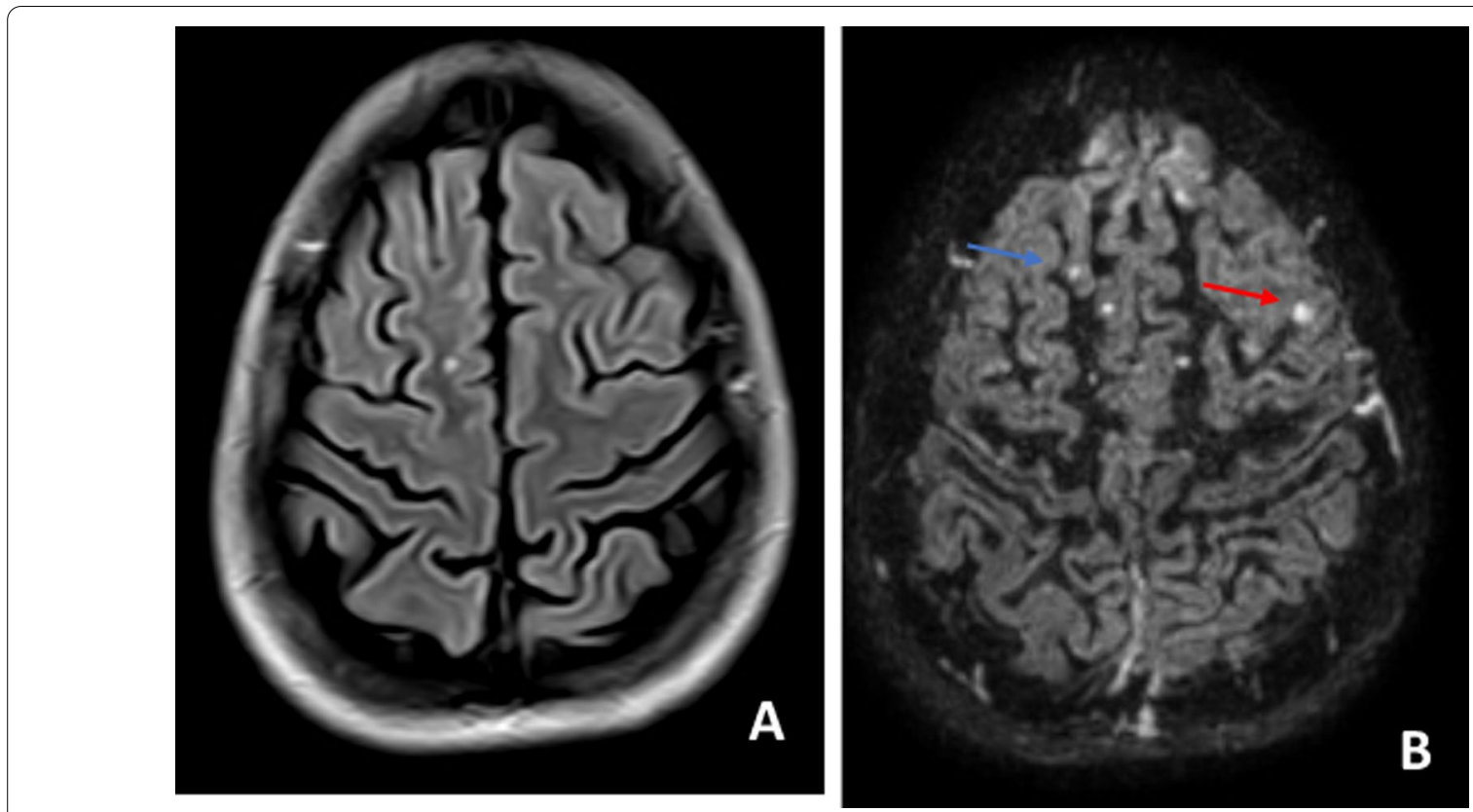

Fig. 1 A 28-year-old female patient with secondary progressive MS and EDSS score of 3. A Axial FLAIR and B axial DIR at the same level revealed multiple cortical and subcortical plaques, the axial DIR revealed cortical plaque-type 1 (red arrow) and type 2 (blue arrow) which was not identified in the FALIR sequence
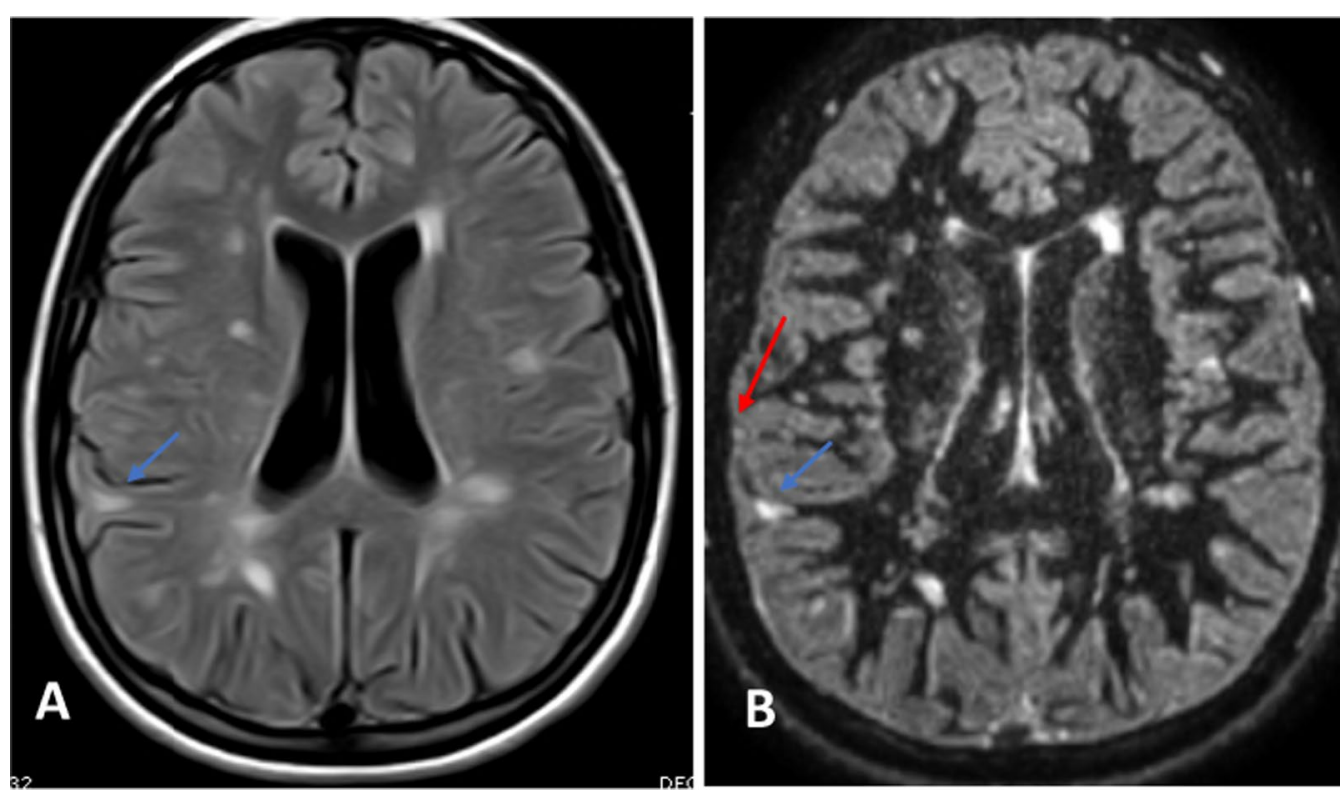

Fig. 2 A 35-year-old female patient with relapsing-remitting MS and EDSS score of 2.5. A Axial FLAIR revealed right parietal cortical/juxtacortical plaques (blue arrow) and multiple periventricular, deep white matter and cortical plaques. B Axial DIR at the same level clearly define the right parietal plaque as juxtacortical plaques, it also revealed type 3 cortical plaques (red arrow) which were seen only in the DIR, and multiple periventricular, deep white matter and cortical plaques are also noted

The current study was conducted over eighty-two people with a mean age of $34.5+8.12$ years; of which 60 patients were females. According to the type of MS at first diagnosis, there were 59 cases of relapsing-remitting MS, 5 cases of secondary progressive MS, 4 cases of primary progressive MS, 8 clinically isolated syndrome 


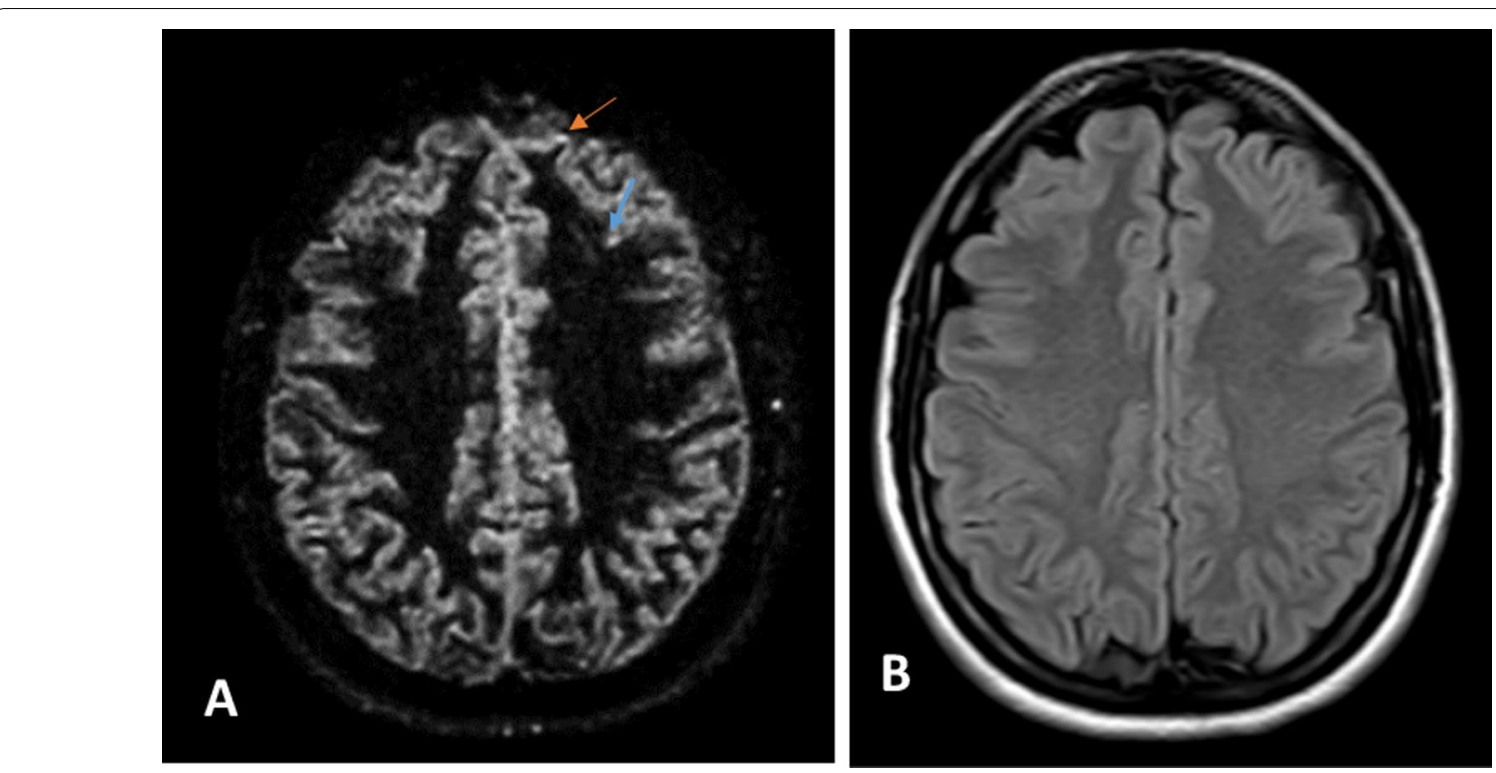

Fig. 3 A 29-year-old female patient with relapsing-remitting MS and EDSS score of 2. A Axial DIR clearly define the left frontal type 2 (blue arrow) and type 3 (orange arrow) cortical plaques. B Axial FLAIR at the same level didn't reveal the lesions identified on the DIR
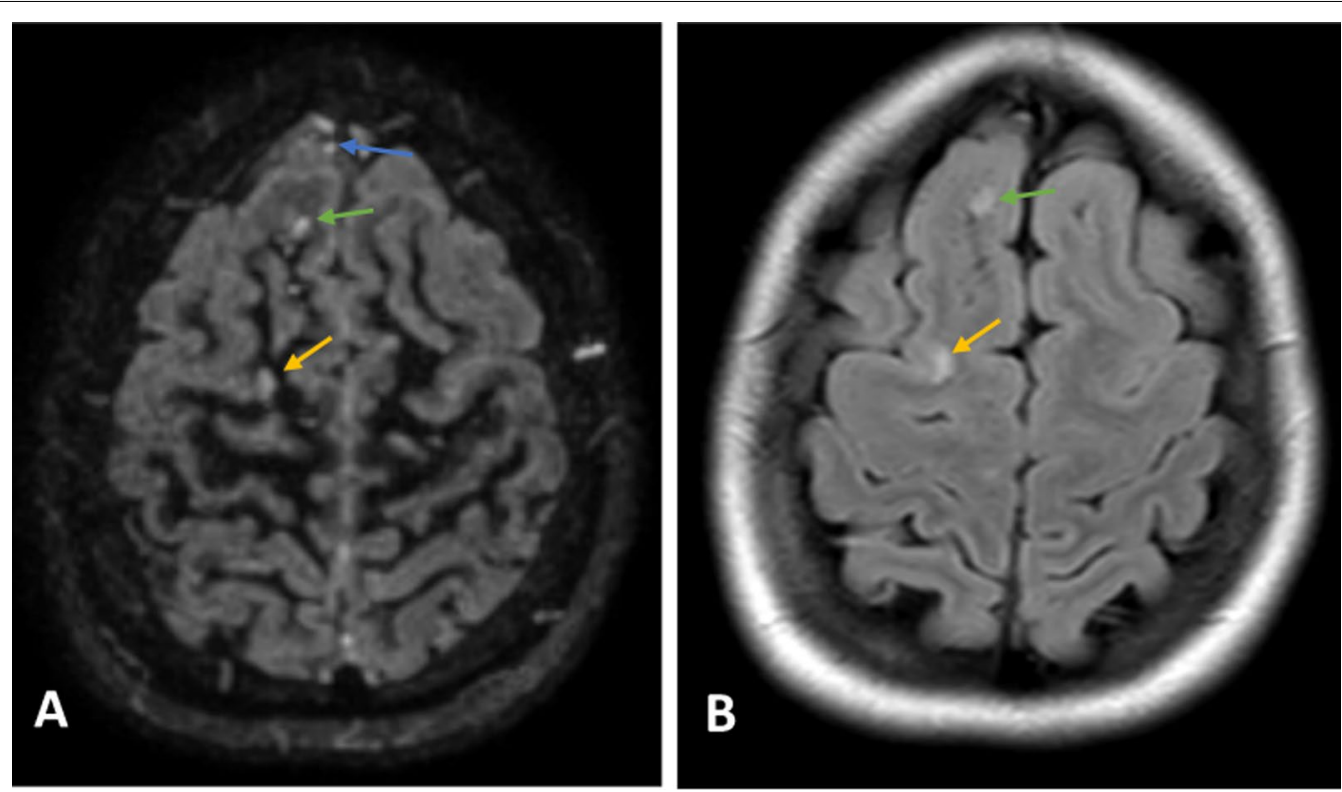

Fig. 4 A 31-year-old female patient with relapsing-remitting MS and EDSS score of 3. A Axial DIR revealed right frontal type 1 (orang arrow), type 2 (green arrow) and type 3 plaque (blue arrow) cortical plaques. B Axial FLAIR at the same level defines the type 1 and 2 cortical plaques but didn't reveal the type 3 cortical plaque

cases and 6 radiologically isolated syndrome cases. A study by Hamed et al. [8] included 90 patients with a mean age of $33.24+-8.93$, of which the majority were relapsing-remitting multiple sclerosis cases (91.1\%).

DIR suppresses the signal of both CSF and white matter allowing much better contrast between both gray and white matter with consequent superior delineation of the gray matter and its cortical subtypes. FLAIR sequence, on the other hand, cannot exactly or clearly show the border between the cortex and subcortical white matter. Type I lesions extend through both the white matter and the gray matter, type II lesions are entirely located within 

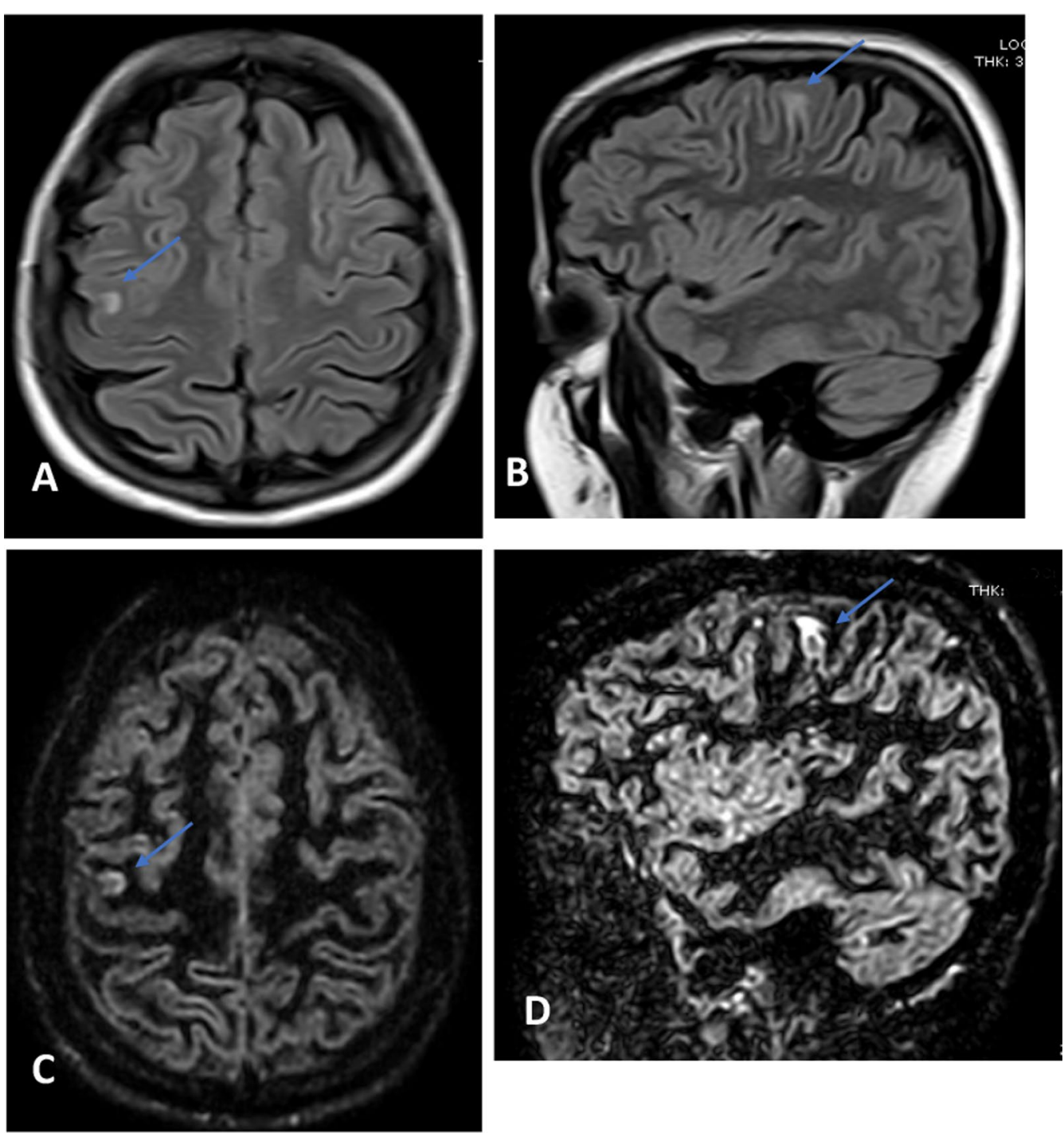

Fig. 5 A 38-year-old female patient with relapsing-remitting MS and EDSS score of 2. A, B Axial and sagittal FLAIR revealed right cortical worm-like type 4 (blue arrow) and two subcortical plaques. C, D Axial and sagittal DIR at the same level clearly defines the right cortical type 4 plaque and the subcortical plaques (blue arrow)

the cerebral cortex, do not make contact with the subcortical white matter or the pia mater and are small. Type III lesions are subpial in location, usually not extending beyond layers 3 and 4 of the cortex. finally, type IV lesions extend over the entire width of the cortex and do not extend into the subcortical white matter and might cover several gyri or entire lobes [12].

In this study, the number of cortical plaques types $1,2,3$ and 4 detected by 3D DIR sequence was significantly more than the FLAIR sequence with $185 \%$, $234 \%, 258 \%$ and $152 \%$ percent improvement $(p<0.001)$. The current study results were similar to that of
Guerts et al. [13] who showed that the DIR sequence resulted in a $152 \%$ increase in the cortical lesion detection rate. Elnekeidy et al. [14] and Vural et al. [15] also reported the highest accuracy of the DIR in the detection of intracortical lesions. A study by Ertan et al. [16] acknowledged that the 3D DIR sequence detected intracortical lesions five times more than that of the 3D FLAIR sequence. In a study by Calabese et al. [17], several patients presented only with 'Cortical onset' of MS. Pirko et al. [18] also reported that many MRI studies have shown that gray matter pathology is already present at the clinical onset of the disease, independent of white matter pathology which is subtle or very limited. 

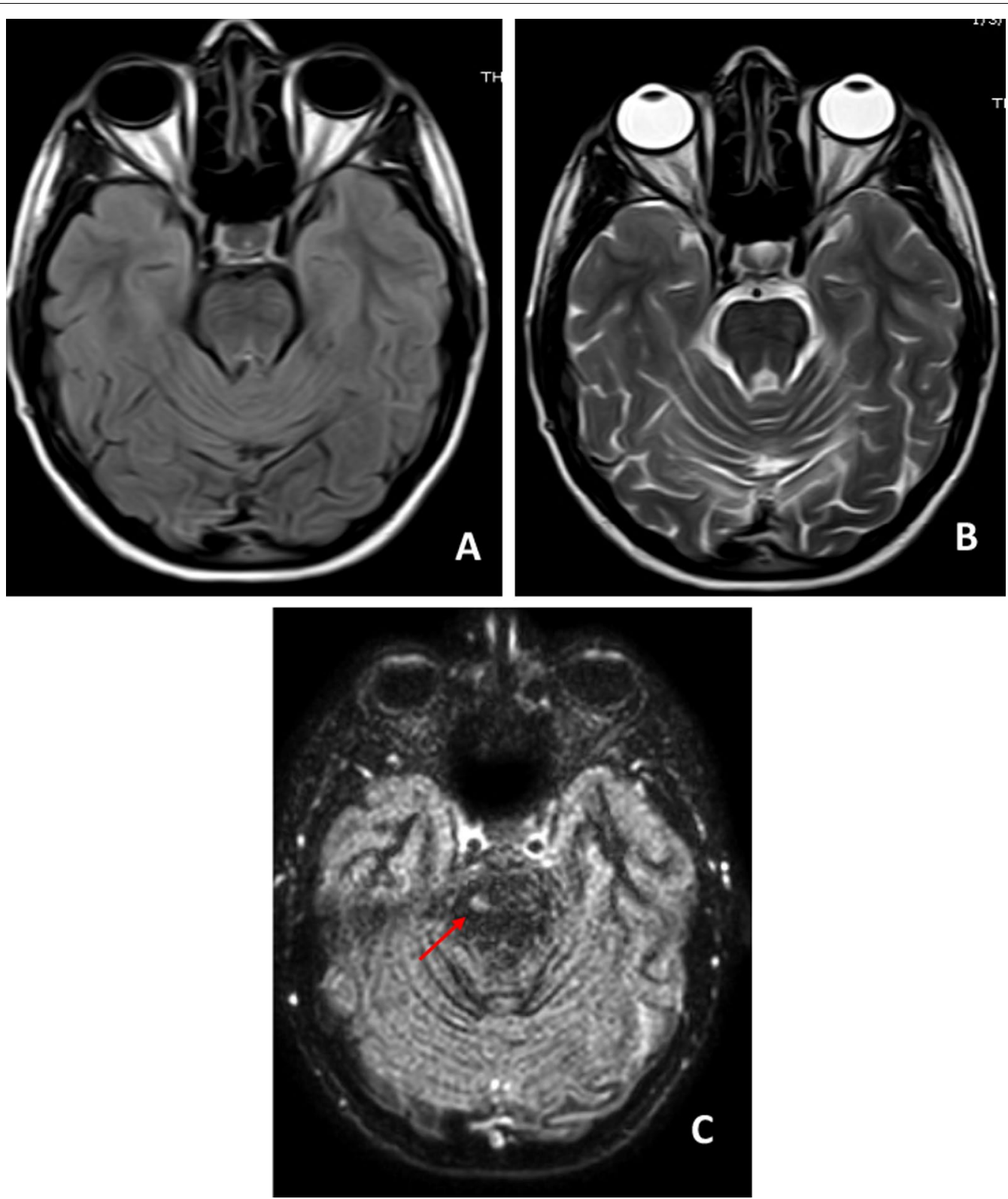

Fig. 6 A 21-year-old female patient with relapsing-remitting MS and EDSS score of 1.5. No evidence of MS plaque in the axial FLAIR (A) and T2 WI (B). C Axial DIR at the same level clearly defines the pontine plaque (red arrow)

3D DIR in this study was superior in the detection of all overall cortical types as well as juxtacortical, juxtacortical extended MS plaques with 204\%, 119\% and $169 \%$ plaque gain in comparison to FLAIR $(p=<0.001$, 0.010 and $<0.001$, respectively). In a study by Ertan et al. [16], DIR detected 1.76 times more JX lesions than FLAIR which was not significant statistically, Guerts et al. [13] also showed more JX lesions on DIR. On the other side, Moraal et al. [19] and Vural et al. [15] reported a higher number of juxtacortical lesions with FLAIR than DIR sequence, yet both studies showed no significant difference.

3D DIR was significantly better than FLAIR for MS plaque number detection in the periventricular white matter, subcortical white matter, infratentorial region and the overall MS plaques burden with a relative improvement of $115 \%, 112 \%, 131 \%$ and $122 \%$, respectively $(p<0.001,0.038,<0.001,<0.001$, respectively). Different studies as with this study showed that the DIR 
Table 3 Correlation between duration of disease, EDSS and the number of attacks per year and the number of MS plaques in different anatomical regions

\begin{tabular}{|c|c|c|c|c|c|c|}
\hline \multirow[t]{2}{*}{ MS plaque location } & \multicolumn{2}{|c|}{ Duration } & \multicolumn{2}{|l|}{ EDSS } & \multicolumn{2}{|c|}{ Attacks per year } \\
\hline & $\rho^{+}$ & $p$ value & $\rho^{+}$ & $p$ value & $\rho^{+}$ & $p$ value \\
\hline Cortical type I & 0.260 & $0.018^{*}$ & 0.356 & $0.001^{*}$ & 0.154 & 0.167 \\
\hline Cortical type II & 0.244 & $0.027^{*}$ & 0.197 & 0.076 & 0.291 & $0.008^{*}$ \\
\hline Cortical type III & 0.354 & $0.001^{*}$ & 0.260 & $0.018^{*}$ & 0.269 & $0.014^{*}$ \\
\hline Cortical type IV & -0.007 & 0.952 & 0.016 & 0.889 & -0.033 & 0.769 \\
\hline Overall cortical types & 0.342 & $0.002^{*}$ & 0.368 & $0.001^{*}$ & 0.296 & $0.007^{*}$ \\
\hline Juxtacortical & 0.258 & $0.019^{*}$ & 0.134 & 0.229 & 0.280 & $0.011^{*}$ \\
\hline Juxtacortical expanded & 0.379 & $<0.001^{*}$ & 0.343 & $0.002^{*}$ & 0.348 & $0.001^{*}$ \\
\hline Deep gray matter & -0.022 & 0.841 & -0.114 & 0.308 & -0.047 & 0.675 \\
\hline Subcortical WM & 0.240 & $0.030^{*}$ & 0.088 & 0.430 & 0.307 & $0.005^{*}$ \\
\hline Deep WM & 0.594 & $<0.001^{*}$ & 0.183 & 0.099 & 0.478 & $<0.001^{*}$ \\
\hline Periventricular WM & 0.366 & $0.001^{*}$ & 0.281 & $0.010^{*}$ & 0.252 & $0.022^{*}$ \\
\hline Infratentorial & 0.123 & 0.272 & 0.083 & 0.46 & 0.061 & 0.585 \\
\hline Overall MS burden & 0.640 & $<0.001^{*}$ & 0.359 & $0.001^{*}$ & 0.525 & $<0.001^{*}$ \\
\hline
\end{tabular}

+ Spearman correlation

* Significant

was superior in detecting plaques in all these anatomical regions [11, 13-15].

The current study revealed no significant difference between 3D DIR and FLAIR for plaques number detection in deep white matter $(p=0.344)$ and deep gray matter $(p=0.104)$. However, Elnekeidy et al. [14] showed more DWM lesions on the DIR. On the other hand, few other studies found that the FLAIR rather than DIR detected a higher number of lesions in DWM [13, 15, 20]. The contrary outcomes throughout various studies revealed that the DIR and FLAIR have remarkably similar detection rates for the DWM plaques, which could be due to the plaques' location away from the cortical and periventricular regions.

According to a study by Haider et al. [21], the cause and clinical significance of deep gray matter injury in MS remains unknown but their study highlighted the importance of only the caudate nucleus and hypothalamus of which their pathogenic processes included demyelination, neurodegeneration and accumulation of oxidative injury. This study revealed no significant difference between 3D DIR and FLAIR for plaques number detection in the deep gray matter $(p=0.104)$. Ertan et al. [16] and Geurts et al. [13] showed that more lesions were detected by the DIR sequence compared to the FLAIR sequence in the deep GM regions. Ertan et al., however, showed no difference between DIR and FLAIR in the number of lesions in the thalamus.

Hamed et al. [8], Vural et al. [15] and Calabrese et al. [22] observed a positive association of cortical lesion load with the clinical outcome and EDSS of the patient. This study as well showed a significant correlation between MS disease duration, number of attacks and EDSS in relation to the overall cortical MS plaque types, juxtacortical expanded lesions, periventricular white matter lesions and the overall MS plaque burden. On the other hand, no significant correlation was seen for the deep white matter and infratentorial MS plaques. Elkholy et al. [23] showed a positive correlation yet not statistically significant between EDSS and the number of periventricular lesions, deep white matter lesions and infratentorial lesions in both FLAIR and DIR.

We recommended that using artificial DIR, which can create high contrast images from standard input, can detect cortical lesions with high reliability, significantly improving lesion detection in MS patients [5, 24].

\section{Limitations}

The first limitation was the relatively small number of patients. Secondly, cortical lesion volume was not included in this study which would have increased our knowledge of MS pathobiology and disease evolution.

\section{Conclusions}

With little extra time added to the MS MRI brain routine protocol, 3D DIR is a powerful readily available sequence that should be more widely implemented for the diagnosis and assessment of the cortical plaques subtypes and as an indicator for MS degree of disability. 


\section{Abbreviations}

MRI: Magnetic resonance imaging; MS: Multiple sclerosis; CLs: Cortical lesions; DIR: Double inversion recovery; CSF: Cerebrospinal fluid; EDSS: Expanded disability status scale; JX: Juxtacortical lesions; WM: White matter; DWM: Deep white matter; GM: Gray matter.

\section{Acknowledgements}

Not applicable.

\section{Authors' contributions}

AS: Idea of the research, Results, Revising manuscript, correlated the study concept and design. NK: Manuscript writing and editing, Methodology, Collecting cases. MM: Idea of the research, Methodology, Correlated the study concept and design, Writing the manuscript. All authors read and approved the final manuscript.

\section{Funding}

No Funds, sponsorship or financial support to be disclosed.

\section{Availability of data and materials}

The datasets used and/or analyzed during the current study are available from the corresponding author on reasonable request.

\section{Declarations}

\section{Ethics approval and consent to participate}

This study was approved by the Research Ethics Committee of the Faculty of Medicine at Ain shams University in Egypt (FWA 000017585); Reference Number of approval: R35/2020. A written informed consent to participate in this research was given by all patients.

\section{Consent for publication}

All patients included in this research gave written informed consent to publish the data contained within this study.

\section{Competing interests}

The authors declare that they have no competing interest.

Received: 6 December 2021 Accepted: 5 February 2022

Published online: 15 February 2022

\section{References}

1. Chard DT, Griffin CM, Rashid W et al (2004) Progressive grey matter atrophy in clinically early relapsing-remitting multiple sclerosis. Mult Scler 10(4):387-391

2. Calabrese M, Filippi M, Gallo P (2010) Cortical lesions in multiple sclerosis. Nat Rev Neurol 6(8):438-444

3. Herranz E, Giannì C, Louapre C et al (2016) Neuroinflammatory component of gray matter pathology in multiple sclerosis. Ann Neurol 80(5):776-790

4. Thompson AJ, Banwell BL, Barkhof F et al (2018) Diagnosis of multiple sclerosis: 2017 revisions of the McDonald criteria. Lancet Neurol 17(2):162-173

5. Bouman PM, Strijbis VI, Jonkman LE et al (2021) Artificial double inversion recovery images for (juxta)cortical lesion visualization in multiple sclerosis. Mult Scler 14:13524585211029860. https://doi.org/10.1177/13524 585211029860

6. Kolber P, Montag S, Fleischer V et al (2015) Identification of cortical lesions using DIR and FLAIR in early stages of multiple sclerosis. J Neurol 262(6):1473-1482

7. Guzel I, Mungan S, Oztekin ZN et al (2016) Is there an association between the Expanded Disability Status Scale and inflammatory markers in multiple sclerosis? J Chin Med Assoc 79(2):54-57

8. Hamed W, Fathi W, Mahmoud W et al (2019) Diagnostic accuracy of double inversion recovery in delineation of multiple sclerosis lesions and its clinical correlation with expanded disability scoring system. Egypt J Radiol Nucl Med 50:114
9. Bø L, Vedeler CA, Nyland $\mathrm{HI}$ et al (2003) Subpial demyelination in the cerebral cortex of multiple sclerosis patients. J Neuropathol Exp Neurol 62(7):723-732

10. Calabrese M, Battaglini M, Giorgio A et al (2010) Imaging distribution and frequency of cortical lesions in patients with multiple sclerosis. Neurology 75(14):1234-1240

11. Abidi Z, Faeghi F, Mardanshahi Z et al (2017) Assessment of the diagnostic accuracy of double inversion recovery sequence compared with FLAIR and T2W-TSE in detection of cerebral multiple sclerosis lesions. Electron Physician 9(4):4162-4170

12. Kidd D, Barkhof F, McConnell R et al (1999) Cortical lesions in multiple sclerosis. Brain 122(1):17-26

13. Geurts JJ, Bö L, Pouwels PJ et al (2005) Cortical lesions in multiple sclerosis: combined postmortem MR imaging and histopathology. AJNR Am J Neuroradiol 26(3):572-577

14. Elnekeidy AM, Kamal MA, Elfatatry AM et al (2014) Added value of double inversion recovery magnetic resonance sequence in detection of cortical and white matter brain lesions in multiple sclerosis. Egypt J Radiol Nucl Med 45(4):1193-1199

15. Vural G, Keklikoğlu HD, Temel \$̧ et al (2013) Comparison of double inversion recovery and conventional magnetic resonance brain imaging in patients with multiple sclerosis and relations with disease disability. Neuroradiol J 26(2):133-142

16. Ertan G, Ozge A, Ulus S et al (2018) Efficiency of double inversion recovery (DIR) sequence in the evaluation of supratentorial cortical lesions in multiple sclerosis. J Neuroquantol 16(3):23-29

17. Calabrese M, Gallo P (2009) Magnetic resonance evidence of cortical onset of multiple sclerosis. Mult Scler 15(8):933-941

18. Pirko I, Lucchinetti CF, Sriram S et al (2007) Gray matter involvement in multiple sclerosis. Neurology 68(9):634-642

19. Moraal B, Roosendaal SD, Pouwels PJ et al (2008) Multi-contrast, isotropic, single-slab 3D MR imaging in multiple sclerosis. Eur Radiol 18:2311

20. Wattjes MP, Lutterbey GG, Gieseke J et al (2007) Double inversion recovery brain imaging at 3T: diagnostic value in the detection of multiple sclerosis lesions. AJNR Am J Neuroradiol 28(1):54-59

21. Haider L, Simeonidou C, Steinberger G et al (2014) Multiple sclerosis deep grey matter: the relation between demyelination, neurodegeneration, inflammation and iron. J Neurol Neurosurg Psychiatry 85(12):1386-1395

22. Calabrese M, De Stefano N, Atzori MB et al (2007) Detection of cortical inflammatory lesions by double inversion recovery magnetic resonance imaging in patients with multiple sclerosis. Arch Neurol 64(10):1416-1422

23. Elkholy SF, Sabet MA, Mohammad ME et al (2020) Comparative study between double inversion recovery (DIR) and fluid-attenuated inversion recovery (FLAIR) MRI sequences for detection of cerebral lesions in multiple sclerosis. Egypt J Radiol Nucl Med 51:188

24. Finck T, Li H, Grundl L et al (2020) Deep-learning generated synthetic double inversion recovery images improve multiple sclerosis lesion detection. Invest Radiol 55(5):318-323

\section{Publisher's Note}

Springer Nature remains neutral with regard to jurisdictional claims in published maps and institutional affiliations. 